\title{
Inovação e Produtividade em uma Perspectiva Intersetorial: Abordagem Empírica e uma Discussão para o Caso Brasileiro
}

Felipe Queiroz Silva ${ }^{1}$

\section{Resumo}

O objetivo deste artigo é analisar a relação entre inovação e produtividade através de uma resenha da literatura empírica sobre o tema, focando, principalmente, nas metodologias de mensuração e causalidade dessas duas variáveis. Trata-se de uma abordagem em nível da firma e em nível setorial, com a proposição de demonstrar a importância do fluxo de tecnologia e das inovações de produto entre as empresas e entre os setores para o aumento da produtividade. Apresenta-se também alguns indicadores recentes de produtividade e inovação no Brasil à luz de uma recomendação de análise sob o enfoque do fluxo de tecnologia intersetorial.

Palavras-chave: Inovação, Produtividade, Fluxo de Tecnologia Intersetorial

\begin{abstract}
The aim of this paper is to analyze the relationship between innovation and productivity through an empirical review of literature about this theme, focusing mainly on methodologies of measurement and causality of these two variables. The approach used is in the firm level and at the sector level, with the proposition to demonstrate the importance of the technology flow and product innovation between firms and between sectors to understand how this process results on productivity gains. This paper also shows some recent productivity and innovation indicators in Brazil and recommends an analysis under the intersectoral technology flow approach.
\end{abstract}

Keywords: Innovation, Productivity, Intersectoral Technology Flow

JEL: O12; O33; L16; L25

Área 1: Indústria e Competitividade

\footnotetext{
${ }^{1}$ Doutorando em Economia da Indústria e da Tecnologia pelo Instituto de Economia da Universidade Federal do Rio de Janeiro.
} 


\section{Introdução}

O debate econômico sobre os determinantes da produtividade é antigo e ainda longe de se esgotar. Entre os vários estudos e fatores que tiveram essa finalidade, destaca-se, sobretudo, a questão da inovação. Utilizando a definição usual de uma função de produção, no qual o resultado (output) depende dos fatores de produção empregados (inputs), verificase que, quanto maior for a relação entre a quantidade produzida e os fatores utilizados, maior será a produtividade. Espera-se, assim, que quanto mais eficiente uma empresa conseguir empregar os seus fatores, mais produtiva a mesma será. Nesse caso, o aumento da produtividade é um dos objetivos centrais da firma, já que impacta os seus custos de produção, aumentando os lucros e a competitividade. A inovação, com o incremento de novas técnicas e novos produtos, seria um dos fatores essenciais para que esse processo ocorresse.

Tanto na prática quanto na teoria, o procedimento descrito no parágrafo anterior é mais complexo do que se pode supor. Primeiramente, não é tão simples mensurar inovação e produtividade. Numa definição schumpeteriana ${ }^{2}$, a inovação é tratada de forma ampla como novos produtos, novas técnicas, novas fontes de suprimentos e novos tipos de organizações. Nem sempre é possível perceber, dentro do escopo de uma firma, o complexo arranjo de fatores e determinantes do que seria uma inovação, e mais difícil ainda é mensurar, quantificar e comparar os efeitos da mesma não só numa organização, mas no conjunto de um mercado ou na economia como um todo. A mensuração da produtividade também é problemática, e pode diferir substancialmente dependendo da técnica empregada (produtividade do trabalho, produtividade total dos fatores) e das dificuldades de mensuração e separação dos fatores de produção, que também envolvem dotações intangíveis.

$\mathrm{Na}$ literatura econômica, pode-se afirmar que atualmente há um consenso dos autores de várias escolas de que existe de fato uma relação positiva entre inovação e produtividade (ou crescimento econômico). Porém, as formas, causas e hipóteses de como esse processo ocorre e as proposições normativas sobre a inovação podem variar substancialmente. Este aspecto teórico é fundamental por estabelecer não só as bases do objeto de estudo como também as técnicas e formas empíricas de trabalhos sobre o tema. A visão predominante da firma neoclássica como uma função de produção, por exemplo, encaminhou a análise empírica da inovação nesse mesmo sentido. O resultado vem sendo estudos que em sua maioria são insuficientes, pois não se dão conta da complexidade de todo o processo inovativo e das formas de como isso impacta não só uma mesma firma, mas também o seu mercado e outras atividades econômicas.

Analisando a inovação e seu impacto na produtividade como tema central, este trabalho busca fazer uma revisão da literatura empírica sobre o tema, focando, principalmente, nas metodologias de mensuração e causalidade dessas duas variáveis. Tratase mais de uma abordagem em nível da firma e em nível setorial, com a proposição de demonstrar a importância do fluxo de tecnologia entre as empresas e entre os setores para perceber como esse processo resulta em ganhos de produtividade. Apesar de usar algumas referências, não se pretende aqui se debruçar exaustivamente no desenvolvimento teórico e empírico da pesquisa macroeconômica e do crescimento econômico sobre o tema, e nem numa exaustiva abordagem dos determinantes da inovação, mas sim centrado na relação desta última especificamente sobre a produtividade.

\footnotetext{
${ }^{2}$ Schumpeter (1984).
} 
Incluindo esta introdução, o artigo está dividido em cinco seções. Na segunda seção, apresenta-se a abordagem teórica e empírica usual da mensuração da produtividade e da inovação por meio de uma função de produção estendida, bem como suas limitações. Depois, na terceira seção, apresenta-se a abordagem de fluxo de tecnologia intersetorial como uma fonte importante para a compreensão da relação entre inovação e produtividade. Na quarta seção, apresenta-se a evolução recente de indicadores de produtividade e de inovação no Brasil em níveis setoriais e uma interpretação da relação dessas duas variáveis para o caso brasileiro. Por fim, a quinta seção apresenta as considerações finais.

\section{Inovação e produtividade em uma função de produção estendida}

O estudo da relação entre inovação e produtividade claramente se confunde com o programa de pesquisa das teorias do crescimento econômico. Desde Solow (1957), o progresso tecnológico vem sendo destacado pela economia mainstream como o principal fator responsável pelo crescimento econômico agregado. Utilizando a abordagem de uma função de produção típica neoclássica, Solow chegou a conclusão de que quase $90 \%$ do crescimento econômico dos Estados Unidos no pós-guerra foi resultado da combinação de novos recursos, ao invés dos simples acréscimos de mais recursos existentes. Essa abordagem é representada pela equação (1):

$$
Q=A(t) f(X)
$$

Onde Q é o produto (output), X é um vetor de $\mathrm{n}$ inputs $\left(x_{1}, \ldots, x_{n}\right)$, A é considerado um fator de deslocamento que capta o nível de eficiência no processo de produção e $t$ é um índice de tempo. Dado a equação (1), a produtividade da relação entre todos os fatores de produção e o produto, denominado como produtividade total dos fatores (PTF), pode ser obtida através de um simples rearranjo dos termos representado pela equação (2):

$$
P T F=A(t)=Q / f(X)
$$

Na literatura econômica, geralmente se utiliza dois fatores principais de produção (capital e trabalho) em uma função do tipo Cobb-Douglas com hipóteses simplificadoras fortes comuns da teoria neoclássica. Entre essas hipóteses, consideram-se retornos constantes de escala; estrutura de mercado sob competição perfeita; ausência de externalidades (seja da própria tecnologia ou de qualquer outro fator); e, principalmente, a hipótese de que a mudança tecnológica (inovação) é exógena ao modelo. Dessa maneira, a inovação é tratada como um bem público acessível a qualquer firma.

Uma fundamentação microeconômica comumente utilizada ao retratar o impacto da mudança tecnológica sobre a função de produção de uma firma é conhecida na literatura por um esquema de classificação poupadora de fatores (LINK; SIEGEL, 2003). Isso acontece através dos deslocamentos das funções de produção (isoquantas) para a origem em um gráfico que considera o capital $(\mathrm{K})$ e o trabalho (L) como insumos. Esse esquema está representado na Figura 1, no qual uma mudança tecnológica poupadora de trabalho desloca a curva de isoquanta (I) e produto $(\mathrm{Q})$ para o ponto $\mathrm{A}$, enquanto uma mudança poupadora de capital desloca a curva para o ponto B e uma mudança tecnológica neutra desloca a curva I 
para o ponto C. O salto tecnológico altera a eficiência da produção com a utilização de menos trabalho e/ou menos capital, o que diminui os custos e aumenta a produtividade. Nesse esquema é assumido implicitamente que a tecnologia leva a mudanças no processo de produção (novas técnicas), ao invés do acréscimo de novos produtos, já que estes últimos seriam incompatíveis com as hipóteses neoclássicas destacadas no parágrafo anterior ${ }^{3}$.

Figura 1 - Mudança tecnológica poupadora de fatores

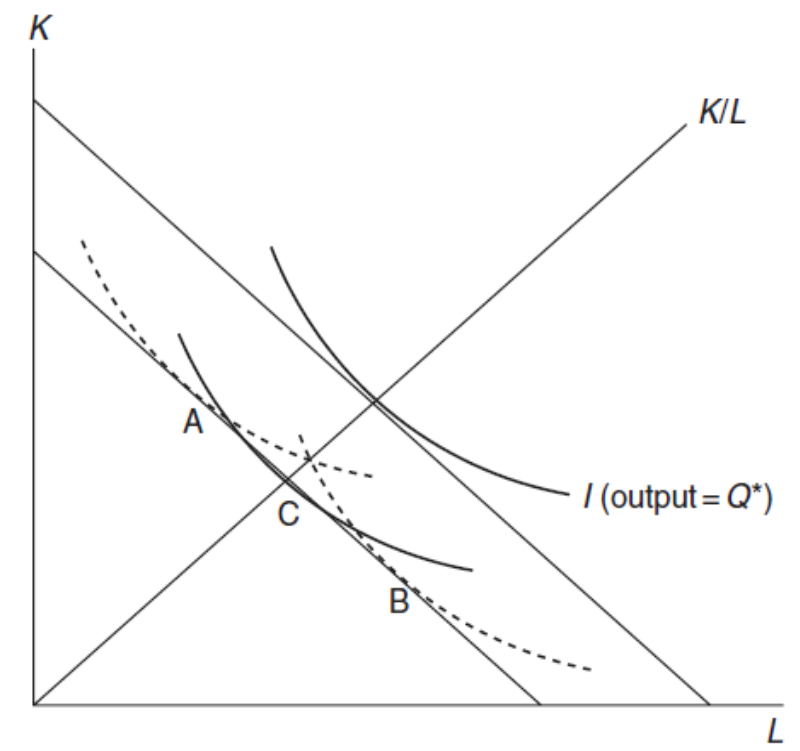

Fonte: Link e Siegel (2003).

Apesar do reconhecimento da teoria neoclássica de que a mudança tecnológica é o fator fundamental para explicar o crescimento da produtividade, claramente suas hipóteses simplificadoras restringiram a complexidade do processo inovativo. Muito dessas simplificações foram superadas pela nova teoria do crescimento endógeno ${ }^{4}$, que passou a reconhecer o caráter endógeno cumulativo da inovação, além de reconhecer mercados imperfeitos e retornos crescentes de escala. Porém, a ideia de equilíbrio e da inovação de processo como poupadora de custos no formato de uma função de produção ainda persiste.

Numa abordagem de economia industrial em nível setorial e em nível de empresas, a análise empírica da relação causal entre inovação e produtividade teve como principal precursor os trabalhos de Zvi Griliches, nos quais são centrados numa abordagem econométrica de estimação da função de produção nos mesmos moldes da perspectiva macroeconômica de Solow (1957). Em um artigo seminal, Griliches (1979) delimita as bases metodológicas para a sua abordagem, que influenciou a maioria dos estudos posteriores. $\mathrm{O}$ autor criou o conceito de estoque de conhecimento da firma, que assim como os fatores de capital e trabalho da função Cobb-Douglas, entraria como um input de produção. Esse fator de estoque de conhecimento tinha como variável proxy principal o investimento em pesquisa e desenvolvimento (P\&D). Dessa forma, Griliches (1979) buscava se afastar das análises de estudos de caso com a finalidade de se concentrar numa abordagem mais geral do impacto da P\&D sobre a produtividade total dos fatores.

\footnotetext{
${ }^{3}$ A incompatibilização da inovação de produto no modelo neoclássico é abordada na seção 3 .

${ }^{4}$ Ver, por exemplo, Aghion e Howitt (1998) e Link e Siegel (2003).
} 
Mais recentemente, com a maior disponibilidade de microdados a partir de surveys de inovação por parte de institutos de pesquisa com base no Manual de Oslo (OCDE, 2005), cresceu bastante o número de estudos que aplicassem os conceitos de Griliches (1979) de forma mais aprofundada. Um modelo muito citado e replicado em outros trabalhos é o do Crépon, Duguet e Mairesse (1998). As implicações dos autores ficaram conhecidas na literatura como Modelo CDM e possuem a principal característica de inserir a hipótese de que a inovação propriamente dita é um processo intermediário entre a decisão e a intensidade de se investir em P\&D e o resultado da produtividade. Dessa maneira, os autores supõem que nem todo esforço inovativo se concretizará de fato no lançamento de inovações bemsucedidas.

Outra característica marcante do modelo é a estrutura de estimações em etapas, bem como técnicas mais robustas com a finalidade de evitar o problema de endogeneidade entre as variáveis de $\mathrm{P} \& \mathrm{D}$, inovação e produtividade. Essa característica é importante, pois muitos trabalhos anteriores possuíam sérios problemas de robustez, já que não se preocupavam em resolver o problema causal da seguinte pergunta: são as empresas inovadoras que possuem maiores níveis de produtividade ou são as firmas mais produtivas que inovavam? Dessa forma, usos de técnicas a partir de modelos de seleção e do uso de variáveis instrumentais foram o foco dessa abordagem.

Algumas evidências empíricas recentes dos estudos sobre inovação e produtividade em nível da firma em vários países, que em sua maioria utilizaram o modelo CDM, foram compiladas e revisadas de forma analítica por Mohnen e Hall (2013). Entres as principais conclusões dos autores estão: i) há uma substancial evidência de um impacto positivo do nível de gasto em P\&D de uma firma sobre a introdução de inovações, seja ela de produto ou processo; ii) no entanto, o impacto da introdução de inovações de produto e processo sobre a produtividade é ambígua e apresenta comportamentos distintos nos vários trabalhos que replicaram o modelo. Na maioria dos trabalhos, não há uma relação estatisticamente significativa da introdução de uma inovação de processo por uma firma sobre a sua produtividade, e, em alguns casos, essa relação chega a ser negativa ${ }^{5}$. Já em outros trabalhos ocorre o oposto, no qual a relação entre inovação de produto e produtividade não apresenta relação causal ${ }^{6}$. Estes resultados são surpreendentes, principalmente no que se refere à inovação de processo. Teoricamente, o aumento da produtividade surge justamente do corte de custos através de técnicas de produção mais eficientes, que numa abordagem neoclássica, por exemplo, deslocarão a função de produção para um nível em que se produza mais com a mesma quantidade de fatores.

Uma hipótese razoável sobre os motivos de não haver um impacto entre inovação de processo e produtividade nesses estudos é de que há problemas de mensuração da inovação nos surveys com base no Manual de Oslo (OCDE, 2005), que não conseguem captar o real efeito da inovação de processo quando colocada junto a inovação de produto, ou simplesmente pela dificuldade de mensuração e coleta desse tipo de dado ${ }^{7}$. Outra hipótese levantada por Mohnen e Hall (2013) é que, como a produtividade geralmente é medida pelo

\footnotetext{
${ }^{5}$ Os autores analisaram mais de 30 trabalhos sobre os efeitos da inovação sobre a produtividade com dados em nível da firma em vários países diferentes.

${ }^{6}$ Por exemplo, Mairesse e Robin (2012) e Lin et al. (2016).

${ }^{7}$ Os surveys de inovação com base no manual de Oslo utilizam de perguntas binárias sobre a introdução de inovação de produto e/ou processo, o que impede uma classificação qualitativa do mesmo. A definição do que seria inovação, em qualquer aspecto, também é problemática e pode incorrer em erro de interpretação pelas empresas respondentes.
} 
valor da receita ou do valor adicionado sobre o número de trabalhadores, a inovação de produto cria um poder de mercado que aumenta a receita. Por outro lado, as melhorias de eficiência das inovações de processo podem não aparecer no valor da receita se essas melhorias resultarem em preços mais baixos sem um aumento correspondente na produção (ao menos no curto prazo).

Esse problema de mensuração da inovação de processo sobre a produtividade já havia sido percebido por Scherer (1984) em um contexto de fluxo de tecnologia entre empresas produtoras e usuárias de inovação. $\mathrm{O}$ autor chamava a atenção que a maior parte dos esforços inovativos de uma empresa são orientados para a criação e melhorias de novos produtos a serem vendido para outras empresas, o que distingue dos esforços de melhorias de processo de produção usados internamente. Os estudos empíricos entre inovação e produtividade focavam e continuam em sua maioria se especializando no impacto da introdução de uma inovação por uma empresa $i$ sobre a produtividade desta mesma empresa $i$, enquanto o importante a ser testado é o impacto da inovação desta firma $i$ para o desempenho da produtividade de uma firma $j$ (SCHERER, 1984).

Scherer (1984) ressalta, porém, que as relações de inovação de produto entre as firmas ou entre setores industrias não são muito claras. Algumas considerações comportamentais e de mensuração devem ser levadas em conta. Pelo lado comportamental, um inovador irá capturar todos os benefícios do aumento da produtividade vinda de novos produtos apenas se ele puder se engajar em descriminação de preço de primeiro grau ${ }^{8}$. Sob uma precificação monopolista, alguns dos benefícios dessa inovação irão necessariamente ser repassadas para os compradores. Se, ainda, a competição for vigorosa, a competição por preço permite também que os inovadores retenham apenas uma pequena parte de suas rendas superiores associadas aos seus novos produtos. Esse aspecto foi primeiramente observado por Griliches (1979), que introduziu o conceito de rent-spillover. Para este autor, as firmas fornecedoras para outras firmas, sob pressão competitiva, geralmente não são capazes de aumentarem os seus preços de acordo com as melhorias qualitativas de seus produtos. Assim, a razão qualidade/preço geralmente aumenta, levando a ganhos de spillover para as firmas que utilizam o produto intermediário ou o bem de capital ${ }^{9}$.

Pelo lado problemático da mensuração, Scherer (1984) salienta que as medidas de produtividade disponíveis, através dos valores de receita, produção ou valor adicionado, não levam em consideração deflatores de índices de preços perfeitamente hedônicos ${ }^{10}$. Como consequência disso, os deflatores de preço comuns subestimam as melhorias de qualidade dos produtos, o que significa que as medidas de produtividade dos inovadores são mais baixas do que seria se índices de preços hedônicos fossem utilizados. Portanto, os ganhos de produtividade mensurados não são observados pelas indústrias que originam a inovação de produto, e sim pelas indústrias à jusante, que utilizam o novo produto em seu processo produtivo.

\footnotetext{
${ }^{8}$ A descriminação de preço de primeiro grau, ou descriminação perfeita, consiste na venda de cada unidade de produto ao preço máximo que o consumidor está disposto a pagar por essa unidade (PINDYCK; RUBINFELD, 2006).

${ }^{9}$ Outro conceito de spillover levantado por Griliches (1979) e de maior dificuldade de mensuração é o knowledge-spillover, que seria induzido pelo conhecimento tácito incorporado.

${ }^{10}$ Preços hedônicos podem ser definidos como bens que são valorizados por seus atributos de utilidade ou por características implícitas, que são revelados aos agentes econômicos a partir de preços observáveis dos produtos diferenciados e ao montante de características específicas em que estão associados (ROSEN, 1974).
} 
Dessa maneira, para determinar os reais efeitos da produtividade dos novos produtos, deve-se traçar o fluxo de tecnologia de uma indústria no qual um novo produto foi originado para as indústrias que utilizam esse produto. Esse pode ser um dos motivos do porquê que as inovações de processo nos trabalhos empíricos econométricos recentes não estão relacionadas com maior produtividade, já que essa relação exige um entendimento em nível interfirma ou intersetorial. O efeito mais importante passa a ser, portanto, na relação entre indústrias produtoras de novos produtos e indústrias usuárias dessas inovações, no qual a inovação é essencialmente de produto, mas utilizada como um novo processo produtivo por outras indústrias. Uma limitação dessa abordagem é de que a mesma apenas é possível em nível setorial por causa da existência de matrizes de insumo-produto, o que é difícil conseguir em nível da firma. A subseção a seguir apresentará os avanços dos trabalhos empíricos sobre essa abordagem.

\section{Fluxo de tecnologia intersetorial e produtividade}

Todas as análises empíricas destacadas na seção anterior partiram da definição de uma função de produção, seja no nível macroeconômico de Solow (1957) ou no nível microeconômico de Griliches (1979). Apesar dos avanços em enfocarem o progresso técnico ou a inovação como determinante principal para o crescimento econômico e para o aumento da produtividade, além de novas especificações (endogeneização da tecnologia) e de novas técnicas econométricas mais robustas, as abordagens se limitam por não expandirem a importância das relações fora dos setores industriais e das firmas no processo inovativo.

Um dos primeiros autores a observar a importância do fluxo de tecnologia intersetorial foi Gustafson (1962) ao questionar alguns trabalhos empíricos de sua época sobre a má especificação dos modelos que tentavam relacionar inovação e produtividade em nível setorial. Para o autor, o gasto em P\&D por uma firma era em sua maioria voltada para a criação de novos produtos, que só apresentariam melhorias de produtividade (redução de custos) para firmas usuárias desses novos produtos. Essa conclusão também foi percebida por Schmookler (1966), que propôs uma solução a esse problema a partir de uma extensão da análise de insumo-produto de Leontief com a incorporação de um fluxo de "invenção". Do ponto de vista conceitual, Schmookler (1966) destacava a importância dos setores usuários das inovações para o desenvolvimento tecnológico ${ }^{11}$. O autor ressaltava que a maior parte da produção das indústrias é direcionada para outras indústrias, e não para o consumidor final. Por essa razão, a habilidade dos produtores de colocarem novos produtos no mercado dependem precisamente da "progressividade" de seus usuários, ou seja, a competência destes é um importante fator em determinar a habilidade do produtor em criar ou melhorar seus produtos.

Nessa relação de interdependência tecnológica setorial, Schmookler (1966) acrescentava ainda que a melhor maneira de melhorar a tecnologia e a técnica de produção de uma indústria é geralmente através da compra de melhorias ou de novos produtos ou

\footnotetext{
${ }^{11}$ No debate sobre os determinantes da inovação, geralmente é ressaltado as diferenças entre as abordagens de inovações induzidos pela demanda (demand-pull) e inovações induzidas pelo impulso da ciência e da tecnologia (technology-push). A hipótese de demand-pull frequentemente é atribuída a Schmookler, que ressaltava não só a demanda final, como, principalmente, os setores usuários ou compradores de novos produtos.
} 
insumos de outras indústrias, ou seja, através da tecnologia incorporada em novos produtos. Para o autor, isso poderia ser medido através de alguma variável de tecnologia em nível setorial (patentes ou gasto em P\&D, por exemplo) em proporção a alguma medida de troca entre os setores industriais. Schmookler (1966) chegou a esboçar uma matriz hipotética de fluxo de tecnologia entre os setores indústrias e que claramente se assemelha com os coeficientes técnicos de Leontief em proporção ao número de patentes. ${ }^{12}$

A ideia de Schmookler (1966) pode ser percebida em Archibugi (1988), que sintetiza que o campo de pesquisa da mensuração da inovação entre setores produtores e usuários deve seguir uma matriz como na Figura 2. A matriz na figura registra as inovações em termos do setor de atividade de produção física i e setores de utilização dessas inovações $\mathrm{k}$. As linhas mostram as indústrias a partir do qual um dado setor transfere tecnologia, já as colunas mostram as indústrias a partir do qual um dado setor adquire tecnologia. A linha y, por outro lado, representa as inovações criadas e utilizadas pela mesma indústria. Esse tipo de análise permite criar índices de dependência e disseminação de tecnologia setorial, tanto do ponto de vista de direção quanto de intensidade. Esse tipo de análise também permite deduzir as interconexões existentes entre indústrias, além de analisar em cada setor àqueles que obtêm e àqueles que fornecem tecnologia.

Figura 2 - Matrix tecnológica entre setores produtores e usuários

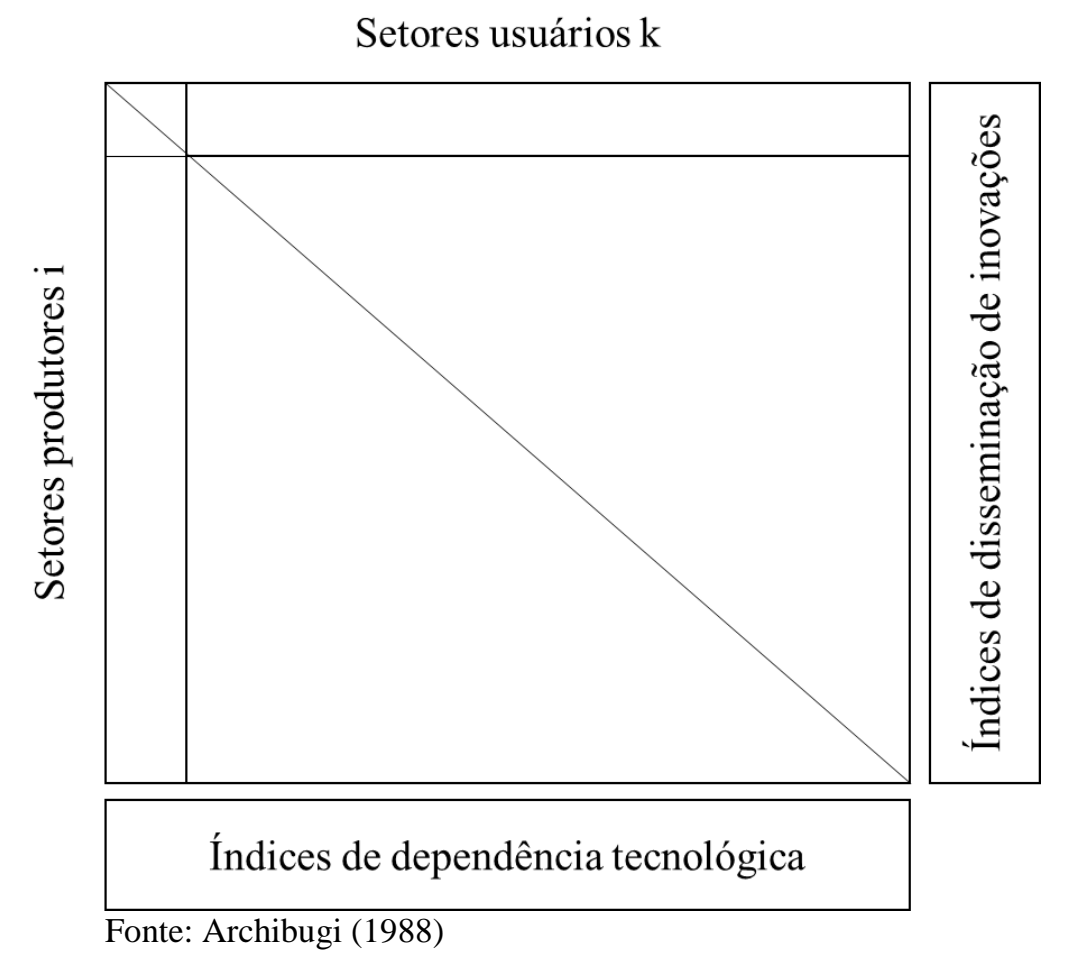

Terleckyj (1980) e Scherer (1982a, 1982b) foram os primeiros autores que testaram a abordagem desenhada por Schmookler (1966) ao atribuírem valores de P\&D e/ou patentes nas matrizes de insumo-produto dos Estados Unidos. Utilizando uma imputação dos valores de P\&D industriais nos coeficientes técnicos da matriz de insumo-produto, Terleckyj (1980)

${ }^{12}$ Schmookler (1966) faleceu antes de executar de forma empírica a sua abordagem, que foi utilizada e citada posteriormente por Terleckyj (1980) e Scherer (1982) (SCHERER, 1984). 
chegou à conclusão de que o retorno do gasto em $\mathrm{P} \& \mathrm{D}$ de um mesmo setor sobre a sua produtividade foi em torno de $29 \%$, enquanto que o retorno do P\&D incorporado através da compra de produtos e insumos de outros setores foi, em média, de $78 \%$, o que evidencia o maior poder explicativo da tecnologia incorporada.

Já Scherer (1982a, 1982b) utilizou um modelo mais complexo e trabalhoso ao também incorporar dados de patentes. $\mathrm{O}$ autor, junto com uma equipe de engenheiros e especialistas, analisou mais de 15 mil patentes e classificou-as entre os setores produtores e usuários de cada uma dessas patentes na matriz de insumo-produto. Ao montar uma matriz de patentes, os gastos em P\&D setoriais foram incorporados nos fluxos estabelecidos. Scherer (1982a) verificou que 75\% das inovações introduzidas nos EUA entre 1964 e 1978 eram de produtos especialmente voltados para outras firmas, e que, assim como em Terleckyj (1980), a produtividade dos setores possuía uma relação com o P\&D incorporado através da aquisição de produtos de outros setores maior do que o P\&D produzido internamente.

A abordagem trabalhosa de Scherer (1982a) foi replicada com os mesmos dados pelo próprio autor anos mais tarde em Scherer (2003), agora utilizando um fluxo de tecnologia intersetorial de maneira mais simples com a incorporação do P\&D setorial sem considerar o trabalho exaustivo de uma matriz de patentes. Os resultados foram muito parecidos entre as duas abordagens, evidenciando que o fluxo de tecnologia intersetorial pode ser utilizado com o uso de técnicas menos dispendiosas.

Paralelamente ao trabalho de Scherer (1982a), Momigliano e Siniscalco (1982, 1984) sugeriram um processo mais sofisticado e rigoroso na construção da matriz de fluxo de tecnologia. Os autores se basearam no conceito de setores verticalmente integrado de Pasinetti (1981). Esse tipo de abordagem explicita tanto os fluxos diretos quanto os indiretos ao também levarem em consideração os componentes da demanda final da matriz de insumoproduto, e forneceram uma figura mais completa de toda a interrelacionalidade tecnológica do sistema de produção. Os autores aplicaram seus conceitos para a economia italiana e perceberam que um pouco mais de $50 \%$ do gasto em P\&D era incorporado em novos produtos. Os autores, porém, estavam mais preocupados com o grau de especialização comercial da Itália, e identificaram uma relação positiva e significativa entre exportações e intensidade tecnológica.

A partir dos trabalhos de Scherer (1982a) e Momigliano e Siniscalco (1984), vários outros autores replicaram os conceitos de fluxo de tecnologia intersetorial com pequenas variações de seus modelos. Entre estes trabalhos, encontram-se os de Griliches e Lichtenberg (1984) e Wolff (1997; 2012), que também utilizaram dados dos EUA, Sterlacchini (1989) e Geroski (1991) com dados do Reino Unido, Goto e Suzuki (1989) com dados do Japão, Hanel (1994) com dados do Canadá e Van Meijl (1997) com dados da França. Todos estes trabalhos encontraram as mesmas conclusões destacados nos parágrafos anteriores: o P\&D incorporado na compra de novos produtos é mais importante para explicar o crescimento da produtividade dos setores industriais do que o $\mathrm{P} \& \mathrm{D}$ próprio desses mesmos setores.

Alguns trabalhos listados no parágrafo anterior também evidenciaram algumas considerações importantes. Geroski (1991), por exemplo, aplicou alguns testes sobre a defasagem temporal dos efeitos do gasto em P\&D intersetorial sobre a produtividade. Nos testes realizados pelo autor, os esforços inovativos realizado em anos anteriores parece impactar mais a produtividade do que os esforços realizados em anos mais recentes. Já Goto e Suzuki (1989) fez alguns testes sobre setores relacionados a tecnologias eletrônicas. Partindo do princípio de que vários setores distintos utilizam tecnologias de base eletrônica (por exemplo: computadores), os autores encontraram que a difusão tecnológica incorporada 
nesses produtos afeta mais outras indústrias relacionadas ao mesmo tipo de tecnologia (por exemplo: máquinas e equipamentos eletrônicos) do que o restante das outras indústrias. Essas considerações ajudam a esclarecer algumas características do processo inovativo, sendo relevante compreender a defasagem temporal dos efeitos da inovação e da proximidade tecnológica dos setores industriais.

\section{Inovação e produtividade no Brasil}

Esta seção apresenta alguns indicadores de inovação e produtividade no Brasil nos anos mais recentes. $\mathrm{O}$ objetivo é não apenas apresentar a evolução dessas variáveis como também o de levantar hipóteses sobre a abordagem de fluxo de tecnologia intersetorial e seus efeitos na produtividade na economia brasileira.

\subsection{Produtividade no Brasil}

Uma das medidas mais usuais de cálculos da produtividade é a produtividade do trabalho, medida como o valor adicionado de uma economia ou de indústrias e empresas específicas sobre o número de trabalhadores ocupados. A produtividade do trabalho é uma medida muito próxima do PIB per capita, métrica usada para auferir o padrão de vida de uma economia. A Figura 3 apresenta esta semelhança por meio da evolução da produtividade do trabalho e do PIB per capita do Brasil entre os anos 1996 e 2014 com valores deflacionados. No entanto, nota-se um descolamento cada vez maior entre as duas variáveis ao longo do tempo. Verifica-se também que a produtividade do trabalho apresentou um crescimento significativo entre 2004 e 2010, mas com taxas cada vez menores a partir da crise mundial de 2008, chegando a apresentar um decréscimo em 2014.

Figura 3 - Produtividade do trabalho e PIB per capita no Brasil, 1996-2014 (1996 = 100)

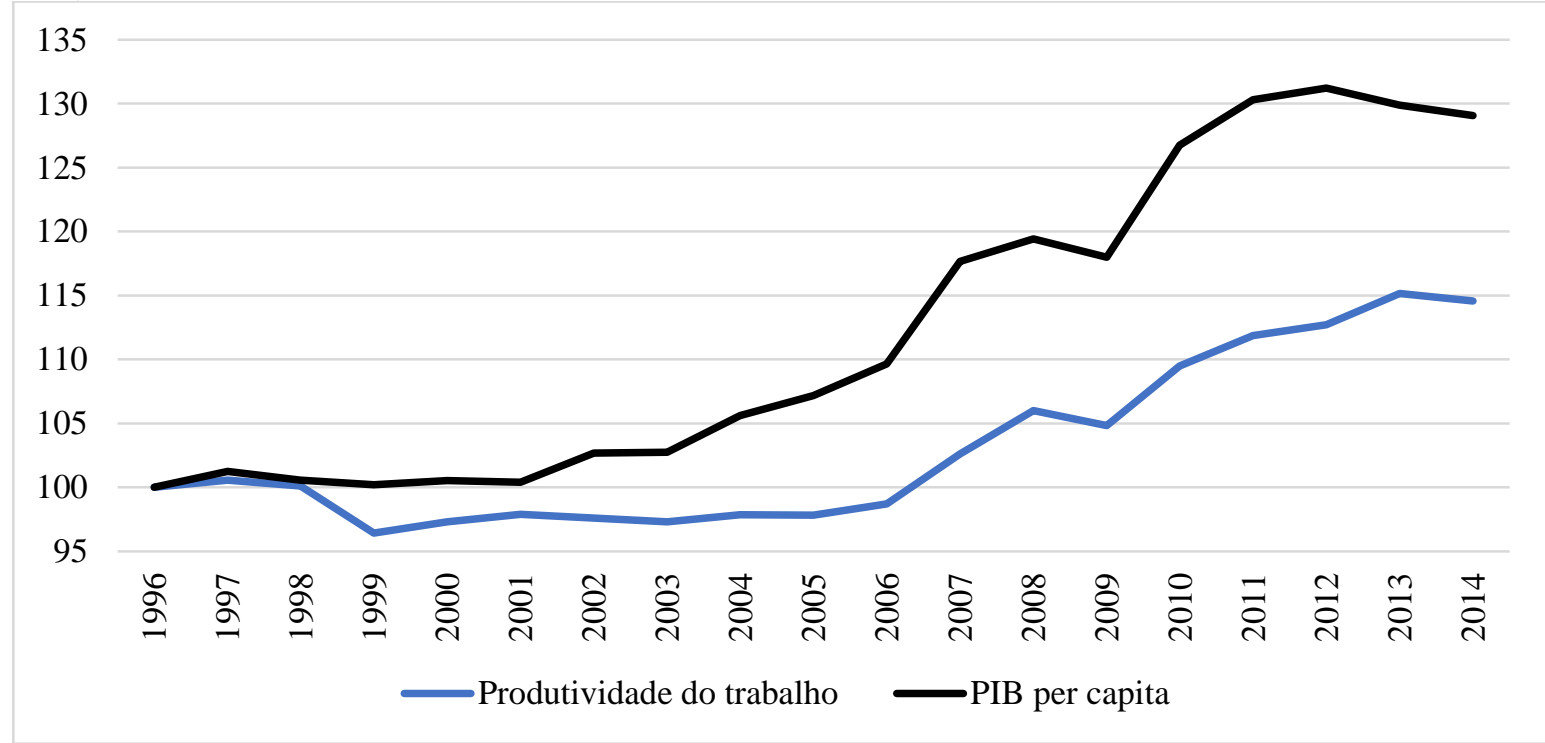

Fonte: Elaboração própria a partir das Contas Nacionais/IBGE. Valores deflacionados. 
Dado que o PIB per capita pode ser decomposto não apenas pela produtividade do trabalho, mas também pela população ocupada sobre o total da população, a Figura 3 mostra que o descolamento cada vez maior entre PIB per capita e produtividade do trabalho se deve mais a aspectos demográficos e de ciclos econômicos. Este fato também vem sendo observado por outros autores. Por exemplo, Cavalcante e De Negri (2014) calculam que $90 \%$ do crescimento da renda per capita entre 1991 e 2001 deveu-se a produtividade do trabalho. Já entre 2001 e 2009, apenas pouco mais da metade foi explicada pelos ganhos de produtividade. O restante foi devido à evolução favorável de variáveis relacionadas ao ciclo econômico (aquecimento do mercado de trabalho) e à demografia. Isso sugere que a redução dos níveis de desemprego e o bônus demográfico explicam uma parcela significativa do crescimento do PIB per capita mais recente na economia brasileira. Como não é possível seguir reduzindo indefinidamente a taxa de desemprego e como os benefícios demográficos estão se exaurindo, não será possível contar com esses fatores no futuro, ou seja, a melhoria do padrão de vida dos brasileiros dependerá cada vez mais de incrementos na produtividade do trabalho.

Outra medida muito usual de produtividade é a produtividade total dos fatores (PTF), que considera não apenas as variações de pessoal ocupado, mas também de outros fatores de produção, como estoque de capital, capacidade instalada e até mesmo capital humano. A Figura 4 apresenta a evolução da PTF calculadas com medidas diferentes por Ellery Jr. (2014) para o Brasil entre 1970 e $2011^{13}$. Em todas as medidas, e apesar das diferenças entre elas, o importante a ser destacado é que, na melhor das hipóteses, se observa um crescimento baixo da produtividade no Brasil nas últimas décadas.

Figura 4 - Trajetória da PTF no Brasil, 1970-2011, diversas medidas (1970 = 100)

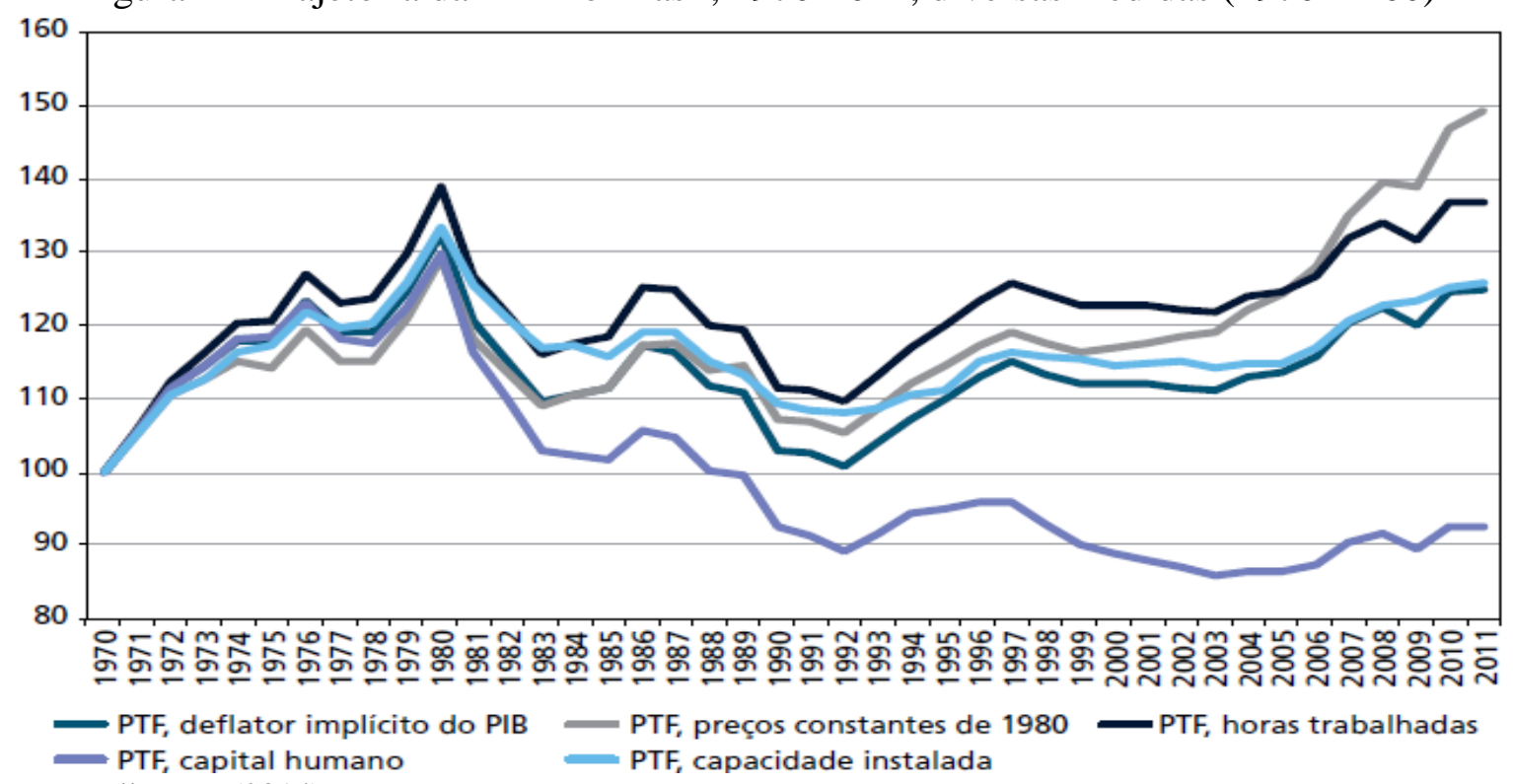

Fonte: Ellery Jr. (2014)

${ }^{13}$ Ellery Jr. (2014) utiliza cinco medidas de produtividade total dos fatores: i) com deflator implícito do PIB; ii) com preços constantes de 1980; iii) utilizando horas trabalhadas ao invés do número de pessoal ocupado; iv) com a utilização do fator de capital humano; e v) com ajuste para utilização de capacidade instalada. Apesar das diferenças nos valores (principalmente quando é utilizado um novo fator como o capital humano, reduzindo o resíduo e consequentemente o valor da PTF), o importante a ser destacado é que o movimento é basicamente o mesmo para os cinco indicadores. 
Em uma análise para níveis setoriais mais desagregados, a Figura 5 apresenta taxas anuais de crescimento da produtividade entre 2000 e 2014 para algumas atividades econômicas no Brasil. Assim como analisado na Figura 3, o total da economia apresenta uma taxa anual de crescimento da produtividade do trabalho significativa entre 2004 e 2008 (2,1\% ao ano). No entanto, verifica-se que, desde a crise mundial em 2008, principalmente a partir de 2010, o Brasil encontra-se em um momento de taxas menores de crescimento e com perspectiva de quedas ainda maiores diante do fraco desempenho econômico nos últimos três anos, no qual atingiu uma taxa anual de crescimento da produtividade do trabalho de apenas $0,3 \%$ entre 2010 e 2014 .

Figura 5 - Taxas médias anuais de crescimento da produtividade do trabalho por atividades econômicas no Brasil, 2000-2014 (\%)

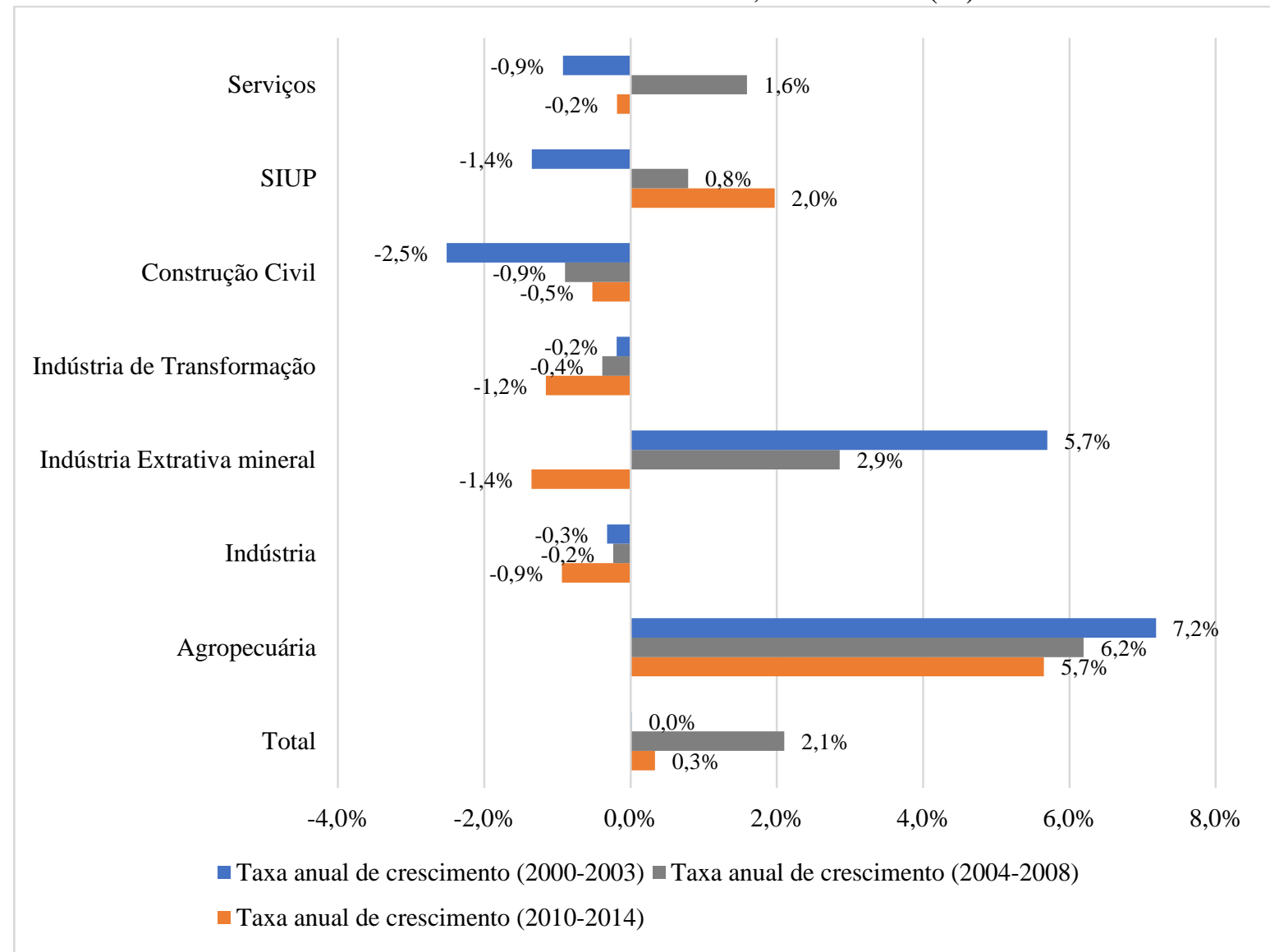

Fonte: Elaboração própria a partir das Contas Nacionais/IBGE. Valores deflacionados setorialmente.

Quando a produtividade do trabalho é desagregada pela agropecuária, indústria (total, extrativa mineral, transformação, construção civil e Serviços Industriais de Utilidade Pública-SIUPs) e serviços, verifica-se, ainda pela Figura 5, o fraco desempenho da indústria total, com taxas médias muito baixas de crescimento e um efeito negativo de $0,9 \%$ entre 2010 e 2014 e de $-1,2 \%$ para a manufatura no mesmo período. Por outro lado, a agropecuária e a indústria extrativa mineral apresentaram um crescimento significativo da produtividade do trabalho nos anos 2000, com exceção para o período mais recente da indústria extrativa em razão da alta queda mais recente do preço das commodities. Já o setor de serviços e os SIUPs apresentaram crescimentos modestos com períodos negativos, enquanto a construção civil 
apresentou um resultado negativo. Dessa maneira, conclui-se que o crescimento verificado da produtividade do trabalho após os anos 2000, ainda que baixo, deveu-se principalmente ao setor agropecuário e à indústria extrativa mineral, uma vez que a indústria de transformação e a construção civil apresentaram desempenhos negativos e o setor de serviços pouco variou.

Figura 6 - Taxas médias anuais de crescimento da produtividade do trabalho por atividades econômicas, 2000-2014 (\%)

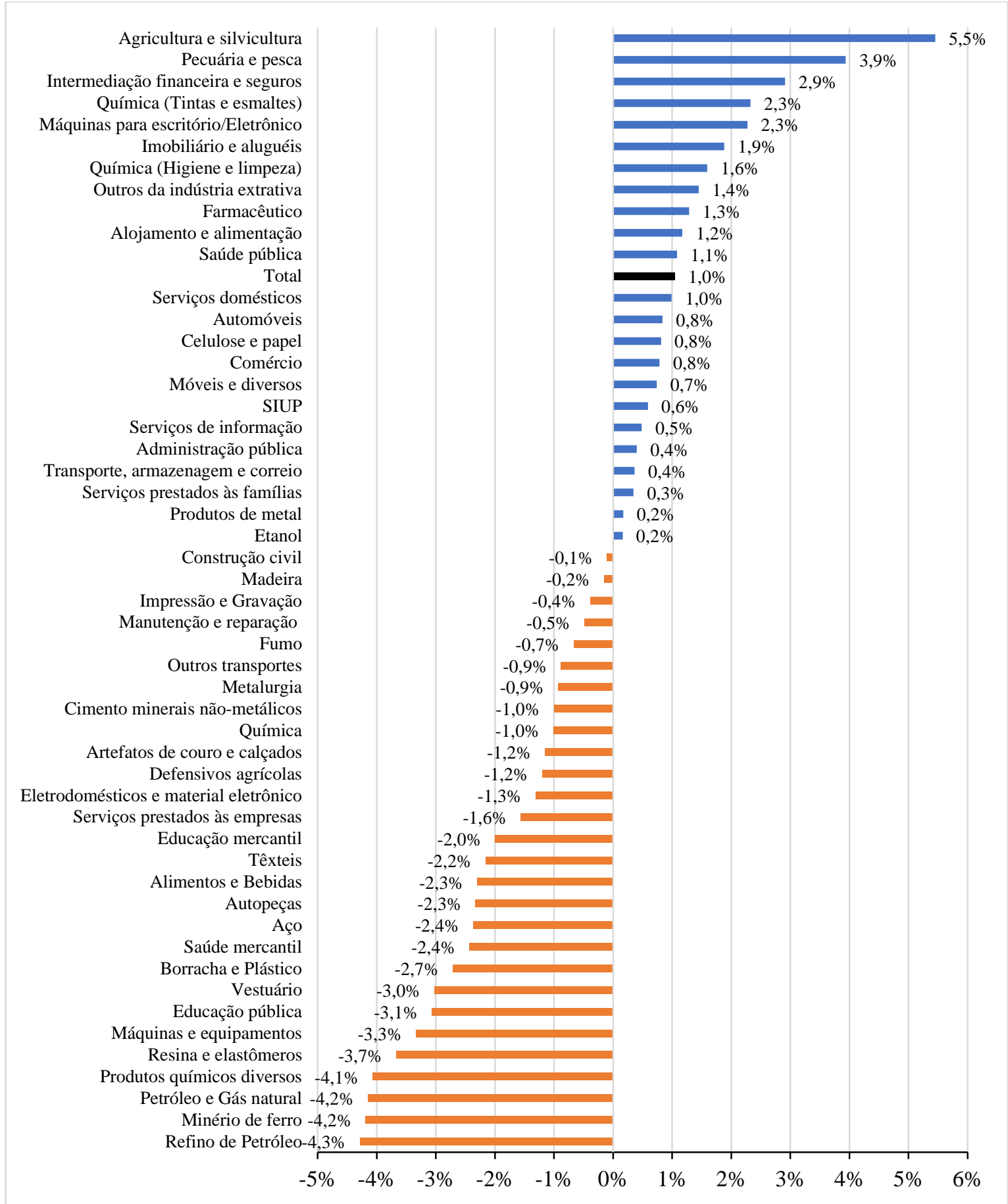

Fonte: Elaboração própria a partir das Contas Nacionais/IBGE. Valores deflacionados setorialmente. 
Para entender melhor quais são as atividades econômicas, em níveis ainda mais desagregados, que afetaram esse resultado, a Figura 6 mostra as taxas médias anuais de crescimento da produtividade do trabalho para alguns setores selecionados no período de 2000 a 2014, último período divulgado e atualizado pela nova metodologia de cálculo do IBGE para esse nível de desagregação. Pela Figura 6, verifica-se que a maioria dos setores da indústria apresentou uma taxa de crescimento negativa da produtividade do trabalho no período analisado. Na manufatura, apenas as indústrias de química básica (tintas e vernizes e higiene e limpeza), máquinas para escritórios e equipamentos eletrônicos, farmacêutica e outros das indústrias extrativas apresentaram taxa de crescimento superior ao total da economia. As indústrias automotivas, celulose e papel, móveis, produtos de metal e etanol também apresentaram taxas positivas, embora os valores sejam pequenos. Como havia sido sinalizado pela Figura 5, a agricultura e a pecuária apresentaram os maiores crescimentos médios anuais no período, enquanto a maior parte dos serviços, com destaque para as intermediações financeiras e de seguros, apresentou taxas positivas

Setores importantes da indústria extrativa, como minério de ferro e petróleo e gás natural, apresentaram taxas de crescimento negativas expressivas no período analisado, ambos com $-4,2 \%$. Isso sugere que o crescimento da indústria extrativa total apresentado na Figura 5 deveu-se a outros produtos, como carvão mineral, minerais não metálicos e minerais metálicos não ferrosos (apresentados como outros da indústria extrativa, com crescimento médio de 1,4\%). Contudo, esse resultado deve ser analisado com cautela, uma vez que a produtividade do trabalho não verifica o ganho de eficiência do capital, o que pode distorcer o resultado devido ao fato de o setor ser altamente intensivo nesse fator. Outra hipótese a ser considerada é que o aumento expressivo da produção desses produtos verificado nesse período, principalmente com o minério de ferro, bem como seu crescente impacto na pauta exportadora brasileira, deu-se mais pelo fator preço do que pelo aumento da eficiência na produção. Como as séries estão deflacionadas, verifica-se que a quantidade de trabalhadores apresentou uma taxa de crescimento maior do que o valor agregado nesses setores.

\subsection{Inovação no Brasil}

O panorama da inovação no Brasil pode ser observado de forma mais aprofundada em nível estatístico desde o ano 2000, com a primeira publicação da Pesquisa de Inovação (Pintec), elaborada pelo IBGE. Em suas seis publicações, a Pintec vem mostrando que a proporção do número de empresas inovadoras vem se mantendo estável. A Tabela 1 apresenta esta proporção estratificada entre indústrias de transformação e indústrias extrativas, segmentos disponíveis pela pesquisa. Para a indústria de transformação, a proporção de empresas inovadoras cresceu de 31,9\% em 2000 para 36,3\% em 2014. Destas, a maioria inovou em processo, mas com uma representação significativa em produto quando comparada com as empresas da indústria extrativa. Estas, por outro lado, com exceção do ano de 2014, inovaram proporcionalmente menos do que a indústria de transformação e também se concentraram no tipo de inovação de processo. 
Tabela 1 - Empresas industriais inovadoras no Brasil, 2000-2014 (\% do total de empresas)

\begin{tabular}{lcccccc}
\hline & $\mathbf{2 0 0 0}$ & $\mathbf{2 0 0 3}$ & $\mathbf{2 0 0 5}$ & $\mathbf{2 0 0 8}$ & $\mathbf{2 0 1 1}$ & $\mathbf{2 0 1 4}$ \\
\hline Empresas Inovadoras & & & & & & \\
$\quad$ Indústrias de transformação & $31,9 \%$ & $33,5 \%$ & $33,6 \%$ & $38,4 \%$ & $35,9 \%$ & $36,3 \%$ \\
$\quad$ Indústrias extrativas & $17,2 \%$ & $22,0 \%$ & $23,1 \%$ & $23,7 \%$ & $19,3 \%$ & $42,0 \%$ \\
Inovadoras em produto & & & & & & \\
$\quad$ Indústrias de transformação & $17,9 \%$ & $20,7 \%$ & $19,8 \%$ & $23,1 \%$ & $17,5 \%$ & $18,4 \%$ \\
$\quad$ Indústrias extrativas & $5,3 \%$ & $6,3 \%$ & $6,4 \%$ & $10,3 \%$ & $5,9 \%$ & $14,3 \%$ \\
Inovadoras em processo & & & & & & \\
$\quad$ Indústrias de transformação & $25,4 \%$ & $27,0 \%$ & $27,0 \%$ & $32,3 \%$ & $32,0 \%$ & $32,5 \%$ \\
$\quad$ Indústrias extrativas & $16,5 \%$ & $20,3 \%$ & $22,3 \%$ & $22,7 \%$ & $18,5 \%$ & $41,5 \%$ \\
\hline
\end{tabular}

Fonte: Elaboração própria a partir da Pintec/IBGE

No entanto, quando se analisa o gasto em atividades inovativas da indústria brasileira, percebe-se que o investimento em $\mathrm{P} \& \mathrm{D}$, seja interno ou externo, é muito pequeno. A Tabela 2 mostra que o valor do $\mathrm{P} \& \mathrm{D}$ interno gasto em proporção da receita líquida de vendas vem se mantendo estável por volta de $0,55 \%$ e $0,68 \%$. A atividade inovativa de maior investimento da indústria brasileira é a aquisição de máquinas e equipamentos, ou seja, as empresas inovadoras brasileiras adquirem proporcionalmente mais conhecimento incorporado na compra de novas máquinas e equipamento do que investem na própria criação de conhecimento $(\mathrm{P} \& \mathrm{D})$.

Tabela 2 - Gastos em Atividades inovativas da indústria no Brasil, 2000-2014 (\% da receita líquida de vendas)

\begin{tabular}{lllllll}
\hline & 2000 & 2003 & 2005 & 2008 & 2011 & 2014 \\
\hline $\begin{array}{l}\text { Aquisição de máquinas e equipamentos } \\
\quad \text { Indústrias de transformação }\end{array}$ & $2,00 \%$ & $1,22 \%$ & $1,34 \%$ & $1,25 \%$ & $1,16 \%$ & $0,86 \%$ \\
$\quad$ Indústrias extrativas & $0,97 \%$ & $1,26 \%$ & $1,38 \%$ & $0,54 \%$ & $0,22 \%$ & $0,70 \%$ \\
$\quad \begin{array}{l}\text { Treinamento de mão de obra } \\
\quad \text { Indústrias de transformação }\end{array}$ & $0,25 \%$ & $0,19 \%$ & $0,19 \%$ & $0,10 \%$ & $0,11 \%$ & $0,10 \%$ \\
$\quad$ Indústrias extrativas & $0,02 \%$ & $0,07 \%$ & $0,02 \%$ & $0,03 \%$ & $0,01 \%$ & $0,01 \%$ \\
P\&D interno & & & & & \\
$\quad$ Indústrias de transformação & $0,65 \%$ & $0,55 \%$ & $0,58 \%$ & $0,64 \%$ & $0,72 \%$ & $0,68 \%$ \\
$\quad$ Indústrias extrativas & $0,23 \%$ & $0,12 \%$ & $0,20 \%$ & $0,13 \%$ & $0,70 \%$ & $0,48 \%$ \\
P\&D externo & & & & & & \\
$\quad$ Indústrias de transformação & $0,11 \%$ & $0,07 \%$ & $0,08 \%$ & $0,10 \%$ & $0,11 \%$ & $0,18 \%$ \\
$\quad$ Indústrias extrativas & $0,05 \%$ & $0,02 \%$ & $0,03 \%$ & $0,02 \%$ & $0,02 \%$ & $0,04 \%$ \\
$\quad$ Outras & $1,06 \%$ & $0,59 \%$ & $0,75 \%$ & $0,56 \%$ & $0,44 \%$ & $0,42 \%$ \\
$\quad$ Indústrias de transformação & $1,00 \%$ & $0,14 \%$ & $0,16 \%$ & $0,15 \%$ & $0,05 \%$ & $0,02 \%$ \\
$\quad$ Indústrias extrativas & & & & & & \\
Total & $3,89 \%$ & $2,48 \%$ & $2,80 \%$ & $2,60 \%$ & $2,46 \%$ & $2,16 \%$ \\
$\quad$ Indústrias de transformação & $1,47 \%$ & $1,61 \%$ & $1,80 \%$ & $0,88 \%$ & $0,70 \%$ & $1,37 \%$ \\
$\quad$ Indústrias extrativas & & & & & &
\end{tabular}

Fonte: Elaboração própria a partir da Pintec/IBGE

A Tabela 3 confirma a importância da aquisição de máquinas e equipamento para a inovação na indústria brasileira. Em 2014, por exemplo, 73,8\% das empresas inovadoras da indústria de transformação e $81,4 \%$ das indústrias extrativas indicaram a aquisição de máquinas e equipamento com grau de importância alta ou média às atividades inovativas. Este número cai, respectivamente, para 15,2\% e 3,8\% na atividade de P\&D interno. Destaca- 
se também a importância da aquisição de software e, principalmente, no treinamento de mãode-obra.

Tabela 3 - Importância alta ou média atribuída às atividades inovativas pelas empresas inovadoras da indústria no Brasil, 2000-2014 (\% do total de empresas inovadoras)

\begin{tabular}{lcccccc}
\hline & 2000 & 2003 & 2005 & 2008 & 2011 & 2014 \\
\hline Aquisição de máquinas e equipamentos & & & & & & \\
$\quad$ Indústrias de transformação & $76,6 \%$ & $80,3 \%$ & $81,3 \%$ & $78,1 \%$ & $75,9 \%$ & $73,8 \%$ \\
$\quad$ Indústrias extrativas & $93,8 \%$ & $78,7 \%$ & $95,7 \%$ & $87,7 \%$ & $81,7 \%$ & $81,4 \%$ \\
Treinamento de mão de obra & & & & & & \\
$\quad$ Indústrias de transformação & $59,1 \%$ & $54,2 \%$ & $59,2 \%$ & $59,4 \%$ & $59,7 \%$ & $61,7 \%$ \\
$\quad \begin{array}{l}\text { Indústrias extrativas } \\
\text { Aquisição de software }\end{array}$ & $63,6 \%$ & $52,1 \%$ & $57,5 \%$ & $87,6 \%$ & $55,0 \%$ & $62,5 \%$ \\
$\quad$ Indústrias de transformação & & & & & & \\
$\quad$ Indústrias extrativas & $\mathrm{ND}$ & $\mathrm{ND}$ & $14,0 \%$ & $24,3 \%$ & $31,6 \%$ & $31,7 \%$ \\
P\&D interno & & & $16,5 \%$ & $13,4 \%$ & $34,9 \%$ & $29,2 \%$ \\
$\quad$ Indústrias de transformação & $34,1 \%$ & $20,7 \%$ & $19,9 \%$ & $11,5 \%$ & $15,9 \%$ & $15,2 \%$ \\
$\quad$ Indústrias extrativas & $26,6 \%$ & $19,0 \%$ & $7,1 \%$ & $20,9 \%$ & $5,5 \%$ & $3,8 \%$ \\
P\&D externo & & & & & & \\
$\quad$ Indústrias de transformação & $4,6 \%$ & $8,2 \%$ & $5,0 \%$ & $4,1 \%$ & $6,4 \%$ & $5,4 \%$ \\
$\quad$ Indústrias extrativas & $16,1 \%$ & $17,1 \%$ & $3,4 \%$ & $3,9 \%$ & $4,8 \%$ & $9,1 \%$ \\
\hline
\end{tabular}

Fonte: Elaboração própria a partir da Pintec/IBGE

Por fim, numa análise da distribuição dos gastos em atividades inovativas por setores industriais, Avellar et al. (2012) também com dados da Pintec mostraram que o padrão setorial está relacionado com o tipo de atividade inovativa empregada. Enquanto setores menos intensivos em tecnologia e dominado por fornecedores como o agronegócio e a manufatura tradicional investiram muito pouco em $P \& D$ interno e utilizaram como principal atividade a compra de máquinas e equipamentos, as indústrias mais intensivas em tecnologia e baseadas em ciências como a eletrônica, insumos de saúde e aeroespacial gastaram uma proporção muito maior em P\&D.

Figura 7 - Distribuição dos gastos em atividades inovativas por sistema produtivo no Brasil, 2005 (\%)

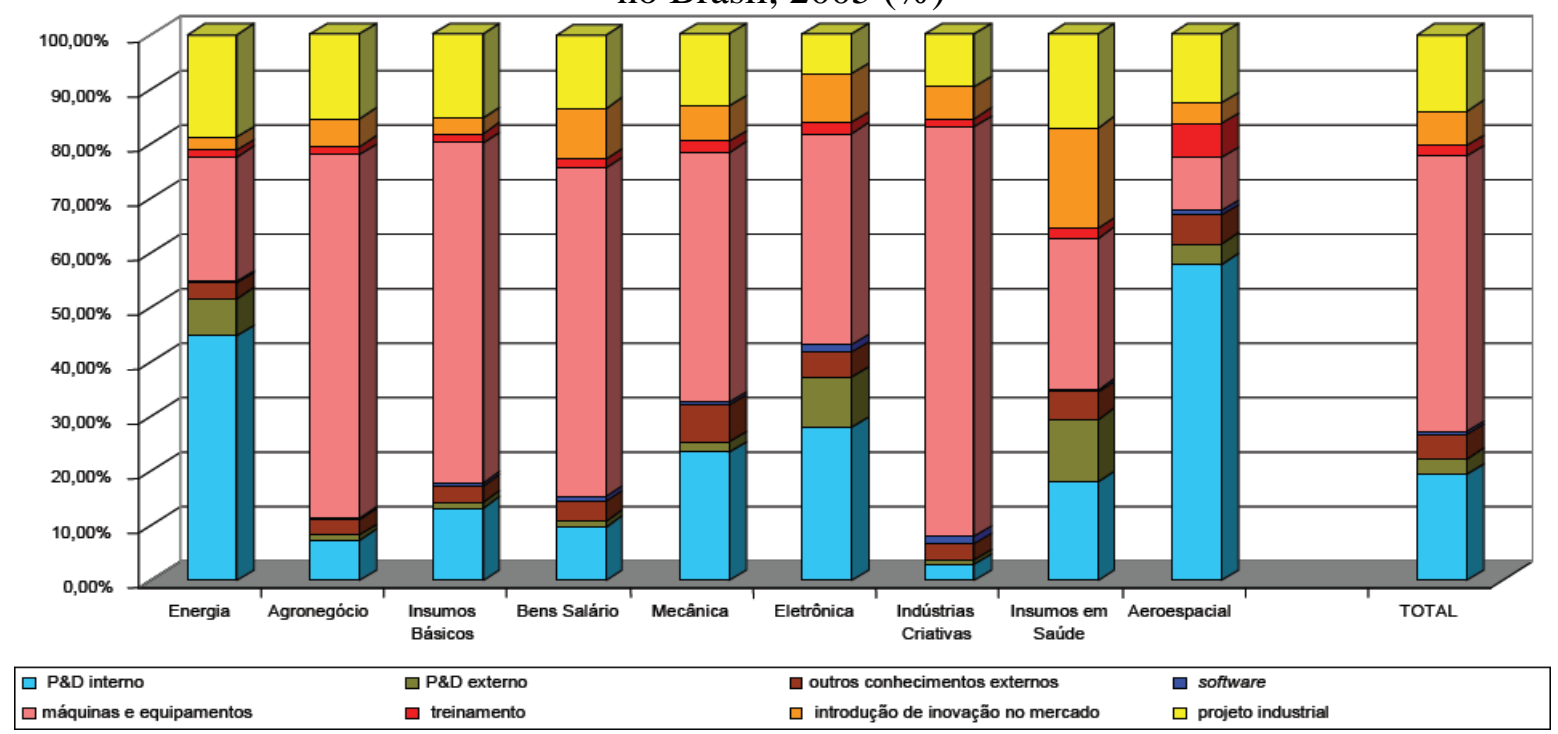

Fonte: Avellar et al. (2012) a partir dos dados da Pintec/IBGE. 
Essas evidências no Brasil estão de acordo com o tipo de análise proposta neste trabalho. Primeiro, percebe-se um baixo crescimento tanto da produtividade quanto da inovação nos últimos anos no Brasil. Segundo, setores menos intensivos em tecnologia como a agricultura e a indústria extrativa foram os que possuíram os maiores níveis de crescimento da produtividade, porém, são os que investiram proporcionalmente menos em P\&D e utilizaram como atividade inovativa principal a compra de máquinas e equipamentos. Não se pode auferir nenhuma relação causal na análise desses trabalhos, mas as evidências sugerem que o crescimento verificado nesses setores específicos se deve mais a inovações (compra de bens de capital) de outros setores da manufatura que desempenharam um crescimento menor da produtividade, ou seja, um aumento da eficiência pela modernização do processo produtivo.

\section{Considerações Finais}

À primeira vista, a relação entre inovação e produtividade parece estar superada no debate econômico. Parece óbvio que o avanço tecnológico aumenta a eficiência do processo produtivo, e de fato aumenta. Porém, a fraca constatação desse fenômeno verificada nos estudos empíricos sugere que o processo é mais complexo e de que muitas abordagens teóricas não são suficientes para explicar a realidade. O forte enfoque teórico nas inovações de processo redutoras de custo acabou desviando a atenção para uma abordagem que considere as inovações de produto e o fluxo tecnológico entre as firmas e entre os setores.

As aplicações de Scherer de uma matriz de fluxo de tecnologia entre empresas e entre setores industriais parece ser o caminho mais razoável para analisar a relação entre inovação e produtividade. Trata-se não apenas de um fato estilizado do capitalismo industrial atual, mas também de uma convergência com a teoria schumpeteriana e neo-schumpeteriana em que o foco principal seja a introdução de novos produtos como propulsor do crescimento econômico, com a destruição criativa de fato, e não apenas uma maior eficiência causada por novos processos.

Dada a complexidade do sistema tecnológico de uma economia e de suas interdependências setoriais, é de supor que boa parte do ganho da produtividade de alguns setores industriais surgiu de inovações ou mudanças tecnológicas proporcionadas por outros setores. A importância e especialização da agropecuária e da indústria extrativa na economia brasileira nos anos mais recentes devem ser analisadas sob um aspecto mais amplo através de suas ligações para trás e para frente na cadeia produtiva. Dessa maneira, evidencia-se a necessidade de uma análise do fluxo de tecnologia intersetorial para buscar compreender as relações entre o processo inovativo e a produtividade no Brasil. Esse tipo de análise é fundamental para sugerir implicações sobre políticas públicas, já que as características setoriais, em termos de externalidades positivas, cumulatividade e sinergias são fundamentais para determinar o desempenho das firmas. Assim, uma política tecnológica deve compreender a complexidade dos fluxos de tecnologia entre as firmas e entre os setores usuários e produtores.

\section{Referências}

AGHION, P.; HOWITT, P. Endogenous Growth Theory. Cambridge: The MIT Press, 1998. 
ARCHIBUGI. D. In search of a useful measure of technological innovation (to make economists happy without discontenting technologists). Technological forecasting and social change, v. 34, pp. 253-277, 1988.

AVELLAR, A. P. M.; BRITTO, J.; STALLIVIERI, F. Capacitação Inovativa e Produtividade na Indústria Brasileira: evidências da diversidade intersetorial. Economia e Sociedade, Campinas, v. 21, n. 2 (45), p. 301-343, 2012.

CAVALCANTE, L. R.; DE NEGRI, F. Evolução recente dos indicadores de produtividade no Brasil. In: DE NEGRI, F.; CAVALCANTE, L. R. Produtividade no Brasil: desempenho. Brasília, DF: IPEA, 2014

CRÉPON, B.; DUGUET, E.; MAIRESSE, J. Research, innovation and productivity: an econometric analysis at the firm level. Economics of innovation and new technology, v. 7, n. 2, p. 115-158, 1998.

ELLERY JR, R. Desafios para o cálculo da produtividade total dos fatores. In. DE NEGRI, F.; CAVALCANTE, L. R. Produtividade no Brasil: Volume 1 - Desempenho. Brasília: ABDI, IPEA, 2014.

GEROSKI, P. Innovation and the Sectoral Sources of UK Productivity Growth. Economic Journal, 101, 1438-1451, 1991.

GOTO, A.; SUZUKI, K. R\&D capital, rate of return on R\&D investment and spillover of R\&D in Japanese manufacturing industries. The Review of Economics and Statistics, v. 71, n. 4, pp. 555-564, 1989.

GRILICHES, Z.; LICHTENBERG, F. Inter-industry technology flows and productivity growth. Review of Economics and Statistics, v. 66, pp. 324-9, 1984.

GRILICHES, Z. Issues in Assessing the Contribution of R\&D to Productivity Growth. Bell Journal of Economics, v. 10, n. 1, p. 92-116, 1979.

GUSTAFSON, W. E. Reserch and Development, new products and productivity change. American Economic Review, v 52, p. 177-185, 1962.

HANEL, P. Interindustry flows of technology: an analysis of the Canadian patent matrix and input-output matrix for 1978-1989. Technovation, v. 14, n. 8, p. 529-548, 1994.

LIN, C-L.; LIN, H-L.; LIN, E. S. Is there a complementary relationship between product and process innovation on productivity in Taiwanese manufacturing firms? Hitotsubashi Journal of Economics, v. 57, n. 2, pp. 139-173, 2016.

LINK, A. N.; SIEGEL, D. S. Technological Change and Economic Performance. London: Routledge, 2003. 
MAIRESSE, J.; ROBIN, S. The importance of process and product innovation for productivity in French manufacturing and services industries. In: ANDERSON, M.; JOHANSSON, B.; KARLSSON, C.; LÖÖF, H. Innovation \& Growth: from R\&D strategies of innovation firms to economy-wide technological change. Oxford: Oxford University Press, 2012.

MOHNEN, P.; HALL, B. H. Innovation and productivity: an update. Eurasian Business Review, v. 3, n. 1, pp. 47-65, 2013.

MOMIGLIANO, F.; SINISCALCO, D. Technology and International Specialization. BNL Quarterly Review, n. 150, pp. 257-284, 1984.

OCDE. Oslo Manual: guidelines for collecting and interpreting innovation data. Paris: OCDE, 2005.

PASINETTI, L. L. Structural change and economic growth: a theoretical essay on the dynamics of the wealth of nations. Cambridge: Cambridge University Press, 1981.

PINDYCK, R. S.; RUBINFELD, D. L. Microeconomia. 6 ed. São Paulo: Pearson Prentice Hall, 2006.

ROSEN, S. Hedonic prices and implicit markets: product differentiation in pure competition. Journal of Political Economy, v 82, n. 1, pp. 34-55, 1974.

SCHMOOKLER, J. Invention and economic growth. Harvard: Harvard University Press, 1966.

SCHERER, F. M. Inter-Industry Technology Flows in the United States, Research Policy, 11, 227-245, 1982a.

SCHERER, F. M. Inter-industry technology flows and productivity growth, Review of Economics and Statistics, v. 64, pp. 627-634, 1982b.

SCHERER, F. M. Using Linked Patent and R\&D Data to Measure Inter-Industry Technology Flows. In: GRILICHES, Z. R\&D, Patents, and Productivity, Chicago, University of Chicago Press, 1984.

SCHERER, F. M. Technology Flows Matrix Estimation Revisited, Economic Systems Research, v. 15, n 3, 2003.

SCHUMPETER, J. A. Capitalismo, Socialismo e Democracia. Rio de Janeiro: Zahar, 1984.

SOLOW, R. M. Technical change and the aggregate production function. The Review of Economics and Statistics. v. 39, n. 3, p. 312-320, 1957. 
STERLACCHINI, A. R\&D, innovations, and total factor productivity growth in British manufacturing. Applied Economics, v. 21, pp. 1549-1562, 1989.

TERLECKYJ, N. Direct and indirect effects of industrial research and development on productivity growth of industries. In: KENDRICK, J. W.; VACCARA, B. N. New development in productivity measurement analysis, NBER, Chicago Un. Press: 1980.

VAN MEIJL, H. Measuring intersectoral spillovers: French evidence. Economic Systems Research, v. 9, n. 1, pp. 9-23, 1997.

WOLFF, E. N. Spillover, linkages, and technical change. Economic Systems Research, v. 9, n. 1, pp. 9-23, 1997.

WOLFF, E. N. Spillover, linkages, and productivity growth in the US economy, 1958 to 2007. In: ANDERSSON, M.; JOHANSSON, B.; KARLSSON, C.; LÖÖF, H. Innovation \& Growth. Oxford: Oxford University Press, 2012. 